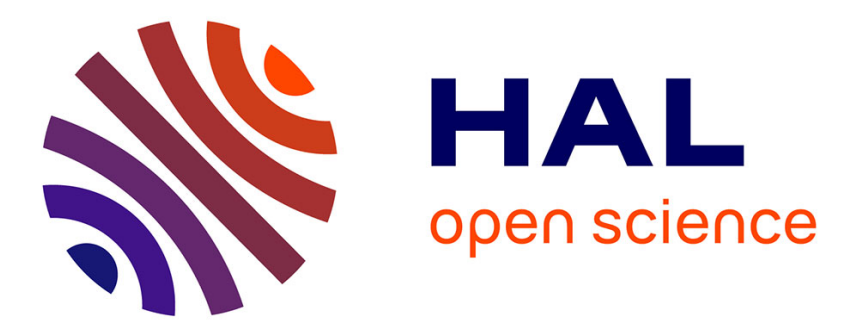

\title{
Oxidative inactivation of ring-cleavage extradiol dioxygenases : mechanism and ferredoxin-mediated reactivation
}

Yves Jouanneau

\section{- To cite this version:}

Yves Jouanneau. Oxidative inactivation of ring-cleavage extradiol dioxygenases: mechanism and ferredoxin-mediated reactivation. K. N. Timmis. Microbiology of hydrocarbons, oils, lipids, and derived compounds, Springer, in press, 2009. hal-00379799

\section{HAL Id: hal-00379799 \\ https://hal.science/hal-00379799}

Submitted on 29 Apr 2009

HAL is a multi-disciplinary open access archive for the deposit and dissemination of scientific research documents, whether they are published or not. The documents may come from teaching and research institutions in France or abroad, or from public or private research centers.
L'archive ouverte pluridisciplinaire HAL, est destinée au dépôt et à la diffusion de documents scientifiques de niveau recherche, publiés ou non, émanant des établissements d'enseignement et de recherche français ou étrangers, des laboratoires publics ou privés. 
Oxidative inactivation of ring-cleavage extradiol dioxygenases : mechanism and ferredoxin-mediated reactivation

Yves Jouanneau, CEA, iRTSV, LCBM, 38054 Grenoble ; CNRS UMR5249, Grenoble, France. yves.jouanneau@cea.fr

\begin{abstract}
Extradiol dioxygenases are ubiquitous enzymes that catalyze ring cleavage of a wide variety of aromatic compounds. Most of these enzymes contain a ferrous ion at the active site, which is bound to the polypeptide chain through a conserved triad of two histidines and one glutamic acid. During the catalytic cycle, a catecholic substrate first binds at the active site, followed by dioxygen and the ternary complex formed yields a bound superoxide that attacks the substrate, leading eventually to ring cleavage. The active site iron remains ferrous during catalysis, except when poor substrates such as chloro- or methylcatechols are processed. In such cases, the enzyme becomes inactivated through oxidation and eventually loss of its active site iron atom. In Pseudomonas putida mt-2, catechol 2,3-dioxygenase, which is involved in toluene degradation, is inactivated by 4-methylcatechol. The enzyme is however rescued by a specific reactivation system involving a [2Fe-2S] ferredoxin encoded by xylT. The role of this ferredoxin is to reduce the ferric ion of the inactive enzyme thereby regenerating the active catalyst. Recent findings indicate that the electrons needed for the XylT-mediated reactivation are provided by XylZ, a NADH-oxidoreductase that is part of the toluate dioxygenase complex. XylT analogues present in other bacteria have been shown to have a similar role in the reactivation of catechol dioxygenases involved in the degradation of various aromatic hydrocarbons including cresols, chlorobenzene and naphthalene. The occurrence and significance of ferredoxin-mediated extradiol dioxygenae repair systems in bacteria is discussed.
\end{abstract}

\title{
Introduction
}

The cleavage of the aromatic ring is a critical step in the biodegradation of aromatic hydrocarbons by aerobic bacteria. It is catalyzed by two kinds of enzyme, the intradiol and extradiol dioxygenases, which represent two classes of phylogenetically unrelated proteins (Harayama et al. 1992). Although both types of enzymes are active on similar catecholic substrates they have different structures and catalytic mechanisms (Vaillancourt et al. 2006). 
Also, extradiol dioxygenases appear to be more versatile than intradiol dioxygenases as they react with a wider range of substrates and intervene in a greater variety of pathways. However, they are labile enzymes which undergo oxidative inactivation, especially in the presence of poor substrates such as alkyl- or chloro-substituted catechols. Current knowledge of the mechanism underlying extradiol dioxygenase inactivation is reviewed here, in the light of recent reports describing in detail the catalytic cycle of this type of enzyme and the process of mechanism-based enzyme inactivation.

A natural repair system, preventing inactivation of catechol 2,3-dioxygenase, was first discovered in Pseudomonas putida mt-2 carrying the $x y l$ genes responsible for the degradation of toluene and xylene (Polissi and Harayama 1993). The repair system relies on a specific [2Fe-2S] ferredoxin encoded by $x y l T$, which has been shown to reactivate inactivated catechol dioxygenase (Hugo et al. 1998). The properties and mode of action of this ferredoxin are presented below, as well as evidence for the involvement of a reductase in the reactivation process in vivo. The occurrence of XylT-like ferredoxins in other bacteria and their implication in other degradation pathways are discussed.

\section{Oxidative inactivation of extradiol dioxygenases}

Extradiol dioxygenases have been classified into three different subfamilies based on protein sequence analysis (Vaillancourt et al. 2006). Here, only type I enzymes, including the wellcharacterized catechol 2,3-dioxygenase from P. putida mt-2 (C23O $\left.\mathrm{mt}_{2}\right)$ (Kita et al. 1999), and the 2,3-dihydroxybiphenyl 1,2-dioxygenase from Burkholderia xenovorans LB400 (DHBD) (Han et al. 1995) will be considered. These enzymes contain a non-heme FeII ion at the active site, which is bound to the polypeptide chain through two histidines and the carboxylate of one glutamate residue. The FeII ion binds the substrate and molecular oxygen, and directly participates in the ring cleavage reaction which occurs between two carbons adjacent to the diol. On the basis of spectroscopic studies of extradiol dioxygenases, a reaction mechanism for the cleavage reaction has been proposed (Shu et al. 1995). The catalytic cycle starts with the binding of the catecholic substrate to the ferrous ion in a bidentate manner (Fig. 1; I). Substrate binding facilitates the formation of a ternary complex with $\mathrm{O}_{2}$, which gives rise to a semiquinone-superoxo intermediate by transfer of electron density from the substrate to dioxygen mediated by FeII (II). The latter species is thought to attack the substrate to give an alkylperoxo intermediate (III). Then, cleavage of the O-O bond, followed by Criegee rearrangement leads to a lactone intermediate and an FeII-bound hydroxide anion (IV). 
Hydrolysis of the lactone and release of the reaction product complete the reaction cycle. Strong experimental support for this proposed reaction mechanism came from a recent elegant study in which three enzyme-intermediate complexes, including the superoxo (II) and the alkylperoxo (III) states, were trapped, crystallized and structurally characterized (Kovaleva and Lipscomb 2007).

Oxidative inactivation of ring-cleavage extradiol dioxygenases, fig1.TIF

Fig. 1 : Proposed mechanisms for the ring-cleavage reaction and suicide inactivation by extradiol dioxygenases. The scheme is adapted from previous work on the structure of reaction intermediates (Kovaleva and Lipscomb 2007) and mechanism-based inactivation (Vaillancourt et al. 2002) .

During the normal course of the catalytic reaction, there is no evidence that the iron changes redox state. However, when $\mathrm{C}_{2} 3 \mathrm{O}_{\mathrm{mt}-2}$ is incubated with alkylcatechols, enzyme inactivation occurs which primarily results from the accidental oxidation of the active site FeII to FeIII (Cerdan et al. 1994). Analysis of the 4-methylcatechol-inactivated $\mathrm{C}_{2} 3 \mathrm{O}_{\mathrm{mt}-2}$ by EPR spectroscopy revealed that the enzyme contained FeIII at the active site, but the signal obtained was distinct from that of a peroxide-treated enzyme, suggesting that a ligand molecule, possibly 4-methylcathecol or an oxidation product, remained bound to the enzyme (Hugo et al. 1998). Halocatechols such as 3-chlorocatechol also cause suicide inactivation of $\mathrm{C} 23 \mathrm{O}_{\mathrm{mt}-2}$ through a mechanism which has been a matter of debate. It has been proposed that inactivation results either from the oxidation and chelation of the active site iron by the catechol or irreversible covalent modification by an acyl chloride generated during ring cleavage (Bartels et al. 1984). A more recent study showed that DHBD became inactivated during the steady-state cleavage of the catechols, and resulted from an oxidation of the active site iron (Vaillancourt et al. 2002). The substrate was found to increase both the reactivity of the enzyme for oxygen and the rate of $\mathrm{O}_{2}$-dependent inactivation, the latter being faster with poor-substrates like 3-chlorocatechol. In that study, the authors proposed that inactivation of extradiol dioxygenases during turnover involved dissociation of the enzyme-superoxo intermediate at an early step of the catalytic cycle, resulting in the oxidation of the active site iron and release of the substrate (Fig. 1). It has been suggested that substituted catechols promote more rapid inactivation because the substituent hinders the correct positioning of the substrate or of $\mathrm{O}_{2}$ at the active site, thus slowing down the catalytic reaction and favoring abortion of the catalytic cycle. Consistent with this idea, it was found that ortho-chlorinated dihydroxybiphenyls, which promote suicide inactivation of DHBD, bind the enzyme active site in such a position that the chlorine atom might affect the binding of $\mathrm{O}_{2}$ (Dai et al. 2002). 
Interestingly, a catechol dioxygenase showing high sequence similarity with $\mathrm{C}_{2} 3 \mathrm{O}_{\mathrm{mt}-2}$ was found to efficiently cleave 3-chlorocatechol (Kaschabek et al. 1998). This enzyme allowed $P$. putida GJ31, the strain from which it was isolated, to metabolize both toluene and chlorobenzene via the meta cleavage pathway (Mars et al. 1997). Although the structural features that makes this enzyme resistant to inactivation have not been determined, one can assume that residues involved in substrate binding play an essential role in the mechanism of resistance. Supporting this idea, the activity of the extradiol dioxygenase from Sphingomonas xenophaga BN6 towards 3-chlorocatechol was enhanced in variants of the enzyme carrying amino acid replacements in a protein region thought to be involved in substrate binding (Riegert et al. 2001). It is to note that the enzyme from strain BN6 is slightly different from the GJ31 enzyme in that the former converts 3-chlorocatechol to chloromuconic semialdehyde whereas the latter produces 2-hydroxymuconate.

\section{A natural repair system dedicated to the reactivation of catechol 2,3-dioxygenase}

In $P$. putida mt-2, the TOL plasmid carries a cluster of genes responsible for the complete degradation of toluene. The $x y l$ genes are organized in two operons, one of which encodes the enzymes that convert toluene to benzoate, the other enzymes that transform benzoate into central metabolites. The latter operon contains 13 genes, including the $x y l E$ gene encoding $\mathrm{C} 23 \mathrm{O}_{\mathrm{mt}-2}$, which is immediately preceded by $x y l T$, a gene coding for a $[2 \mathrm{Fe}-2 \mathrm{~S}]$ ferredoxin (Harayama and Rekik 1990). A xylT-deleted mutant was found to lose the ability to grow on $p$-xylene and $p$-toluate, due to the irreversible inactivation of $\mathrm{C} 23 \mathrm{O}_{\mathrm{mt}-2}$ by 4 -methylcatechol (Polissi and Harayama 1993). Hence, in preventing inactivation of the catechol dioxygenase, XylT allowed strain mt-2 to grow on para-substituted alkylbenzenes, thus extending the range of substrates utilized by the bacteria. The mechanism of action of XylT was studied after purification of the protein overproduced in E. coli (Hugo et al. 1998). XylT appeared to be a [2Fe-2S] ferredoxin, showing some similarities to plant counterparts, but also with distinctive biochemical and spectroscopic properties. As predicted from the prevalence of basic residues in its sequence, XylT has an isoelectric point of 8.27 . This basic character shared by XylT analogues is a unique feature among ferredoxins and might be of critical importance for its interaction with $\mathrm{C}_{2} 3 \mathrm{O}_{\mathrm{mt}-2}$ (see below). XylT also exhibited an unusual instability upon exposure to air oxygen or upon incubation at moderate temperature, with half-lives of 69 min at $25^{\circ} \mathrm{C}$ in air and $70 \mathrm{~min}$ under argon at $37^{\circ} \mathrm{C}$. This instability, which resulted in the loss of the Fe-S cluster, might reflect a high degree of exposure of the cluster to the solvent, as 
suggested from changes in the EPR signal of the protein in the presence of glycerol (Hugo et al. 1998).

The direct role of XylT in $\mathrm{C}_{2} 3 \mathrm{O}_{\mathrm{mt}-2}$ reactivation was assessed in in vitro experiments where the purified ferredoxin was incubated with the inactivated catechol 2,3-dioxygenase and 5deazaflavin as a source of reductant. Results showed that XylT mediated a rapid recovery of the dioxygenase activity in a reaction that was dependent on reductant supply. When XylT was replaced by another [2Fe-2S] ferredoxin with similar redox properties, like spinach ferredoxin, no reactivation occurred. Hence, the reactivation was XylT-specific and likely proceeded through a reduction of the active site iron of the dioxygenase. To assess this assumption, the redox state of iron at the catalytic site was monitored by EPR spectroscopy upon reactivation. Results showed that the 4-methylcatechol-inactivated $\mathrm{C}_{2} 3 \mathrm{O}_{\mathrm{mt}-2}$ displayed a signal with a $S=5 / 2$ ground state typical for a ferric ion. Upon reactivation by a catalytic amount of XylT, this signal disappeared, indicating that FeIII was converted to FeII at the active site. Accordingly, the reactivated enzyme treated with nitric oxide yielded a prominent $\mathrm{S}=3 / 2$ EPR signal characteristic of a FeII-NO complex (Hugo et al. 1998). The possibility that reactivation occurred through transfer of ferrous ions from the XylT cluster to the enzyme was ruled out by EPR measurements showing that the integrity of the XylT cluster was unaltered by the reactivation reaction. Taken together, the data provided evidence that XylT mediates $\mathrm{C} 23 \mathrm{O}_{\mathrm{mt}-2}$ reactivation through reduction of the ferric ion at the enzyme active site, as illustrated in figure 2.

In P. putida mt-2, a NAD(P)H reductase probably serves as source of reductant in the XylTmediated reactivation of the dioxygenase. Such an enzyme activity was detected in crude extracts by using a two-step assay involving incubation of a sample of extract with inactive

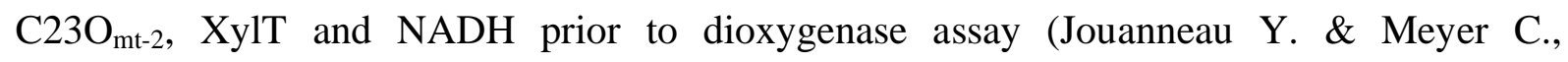
unpublished data). A 43-kDa protein was partially purified, but the instability of this protein precluded further characterization. Nevertheless, the XylT reductase activity was found to be 10-fold lower in a strain lacking the TOL plasmid compared to the wild-type strain mt-2, suggesting that the gene encoding the reductase was plasmid-borne. Analysis of the TOL plasmid sequence indicated that two genes might potentially encode the protein of interest. The two genes were overexpressed in E. coli, but only one of the two gene products proved capable of $\mathrm{C} 23 \mathrm{O}_{\mathrm{mt}-2}$ reactivation in the coupled assay. This protein, encoded by $x y l Z$, was found to be the reductase component of toluate dioxygenase, a two component enzyme that catalyzes the dioxygenation of substituted benzoates (Ge et al. 2002). The $x y l Z$ gene is located in the same operon as $x y l T$ and $x y l E$ indicating that the three genes are co-transcribed. Our 
data provide evidence that the XylZ protein has a dual function, since it acts as a reductase associated with toluene dioxygenase on the one hand, and as a XylT reductase in the specific reactivation of $\mathrm{C} 23 \mathrm{O}_{\mathrm{mt}-2}$, on the other hand (Fig. 2).

Oxidative inactivation of ring-cleavage extradiol dioxygenases, fig2.TIF

Fig; 2 : Ferredoxin-mediated reactivation of catechol 2,3-dioxygenase. $\mathrm{C}_{2} 3 \mathrm{O}_{\mathrm{mt}-2}$ undergoes inactivation upon cleavage of 4-methylcatechol. XylT reductively reactivates $\mathrm{C}_{2} 3 \mathrm{O}_{\mathrm{mt}-2}$, using electrons provided by XylZ, which also serves as reductase for toluate dioxygenase.

\section{Ferredoxin-mediated reactivation of catechol dioxygenases in bacteria degrading other aromatic hydrocarbons}

XylT analogues were found in Pseudomonas strains degrading naphthalene and cresols (Hugo et al. 2000) as well as in Comamonas sp. and Acinetobacter sp. degrading nitrobenzene and aniline, respectively (Tropel et al. 2002). The considered proteins had between 29 and $65 \%$ sequence identity with XylT and were purified as [2Fe-2S] ferredoxins showing similar biochemical and redox properties. Interestingly, all six proteins tested promoted the reactivation of $\mathrm{C}_{2} 3 \mathrm{O}_{\mathrm{mt}-2}$ almost as efficiently as XylT. Moreover, the analogues could functionally replace XylT in vivo, as demonstrated by complementation experiments where the introduction of the $x y l T$-like gene in trans in a $x y l T$ mutant restored the ability of transconjugants to grow on $p$-methylbenzoate (Hugo et al. 2000). Like XylT, the analogues were basic proteins, suggesting that positive charges at the surface of the proteins might be involved in the interaction with catechol dioxygenase. The interactions between XylT, its analogues and $\mathrm{C} 23 \mathrm{O}_{\mathrm{mt}-2}$ were investigated by cross-linking experiments using 1-ethyl-3(3dimethylaminopropyl)carbodiimide (EDC). A 1:1 complex was formed between one subunit of the dioxygenase and one molecule of ferredoxin, even for heterologous pairs of proteins. The formation of covalent complex was affected by ionic strength, indicating that electrostatic forces were involved in the ferredoxin-dioxygenase interactions (Hugo et al. 2000; Tropel et al. 2002). Further studies suggested that the XylT-C23O $\mathrm{O}_{\mathrm{mt}-2}$ complex resulted from the covalent bonding between a primary amine of at least one XylT residue and a carboxylate from one acidic residue of the dioxygenase. Since there are only three lysines in the XylT sequence, replacement of each of the lysyl residues by either a glutamic acid or a glutamine was undertaken by side-directed mutagenesis (Jouanneau Y. and Meyer C., unpublished work). Results showed that variants bearing a single mutation at any of the three lysine positions formed a cross-linked complex with the dioxygenase, whereas two proteins bearing 
a double mutation $(\mathrm{K} 34 \mathrm{Q} / \mathrm{K} 71 \mathrm{E}$ and $\mathrm{K} 34 \mathrm{E} / \mathrm{K} 71 \mathrm{E})$ failed to form a covalent complex with $\mathrm{C}_{23} \mathrm{O}_{\mathrm{mt}-2}$. This indicated that either one of the $\mathrm{K} 34$ or K71 residues of XylT may interact with a corresponding acidic residue of the dioxygenase. Nevertheless, all the constructed variants were found to be competent in $\mathrm{C}_{2} 3 \mathrm{O}_{\mathrm{mt}-2}$ reactivation in vitro, suggesting that electrostatic forces may not be essential for productive interaction between the two partner proteins.

$P$ putida GJ31, a bacterium able to grow on chlorobenzene, contains a chlorocatechol dioxygenase which catalyzes the meta-cleavage of 3-chlorocatechol, in contrast to most other extradiol dioxygenases, which are inactivated by this substituted catechol (Kaschabek et al. 1998). The gene encoding this dioxygenase $(c b z E)$ is preceded by a $x y l T$-like gene called $c b z T$. The same is true for other chlorobenzene-degrading strains using the meta-cleavage pathway (Göbel et al., 2004). The CbzE enzyme was found to undergo inactivation during catalysis of 4-methylcatechol cleavage, suggesting that the loss of activity resulted from an oxidation of the active site iron as shown for $\mathrm{C}_{2} 3 \mathrm{O}_{\mathrm{mt}-2}$. The CbzT gene product was purified as a $[2 \mathrm{Fe}-2 \mathrm{~S}]$ ferredoxin, which efficiently reactivated $\mathrm{CbzE}$ in vitro, most likely by restoring the ferrous redox state at the enzyme catalytic site (Tropel et al. 2002).. Curiously, while three ferredoxin analogues promoted CbzE reactivation, XylT was inefficient and failed to form a covalent complex with CbzE upon incubation with EDC. On the other hand, CbzT was competent in the reactivation of $\mathrm{C}_{2} 3 \mathrm{O}_{\mathrm{mt}-2}$ and could be cross-linked to that enzyme (Tropel et al. 2002).

\section{Occurrence of $x y l T$-like genes in bacterial genomes}

A BLAST search for $x y l T$ analogues in bacterial genomes currently available in the NCBI databases yielded 53 sequences with scores above 32. All sequences share a common 4 cysteine motif for cluster ligation and exhibit a basic character (calculated $\mathrm{pI}>7.5$ ). Most of the retrieved genes were found in the genomes of members of the alpha, beta and gamma subgroups of Proteobacteria, and often lie close to a gene encoding a catechol dioxygenase. In sphingomonads, however, the $x y l T$-like gene is located several $\mathrm{kb}$ away from a $x y l E$ gene, and no $x y l Z$ gene was detected in the large cluster of catabolic genes involved in aromatic and polyaromatic hydrocarbon biodegradation (Pinyakong et al. 2003). Surprisingly, xylT-like genes are also found in bacteria not known for their ability to degrade aromatic compounds, like the nitrogen fixing species Azotobacter vinelandii (GeneBank acc. number EAM07766) and Azoarcus sp. BH72 (GeneBank acc. number CAL95062), the latter strain being a grass endophyte. These strains contain elsewhere in their genomes at least two copies of catechol dioxygenase genes closely related to those involved in alkylphenol degradation. $x y l T$-like 
genes are apparently absent from the bacterial genomes of species belonging to Actinomycetes or Bacillales, numerous members of which are endowed with the ability to degrade aromatic hydrocarbons. For example, Rhodococcus RHA1 has a versatile metabolism and can degrade various aromatic hydrocarbons including polychlorobiphenyls thanks to wide range of catabolic enzymes, including catechol dioxygenases (McLeod et al. 2006). Since such bacteria do not have XylT analogues, they must have evolved alternate strategies to cope with the intrinsic instability of ring-cleavage extradiol dioxygenase. In this respect, it has been observed that DHBD from B. xenovorans LB400, once inactivated by 3-chlorocatechol in vivo, recovered full activity upon removal of the inhibitor. Reactivation took about half an hour, occurred in the presence of an inhibitor of protein synthesis and was independent of a XylT-like ferredoxin (Vaillancourt et al. 2002).

\section{Reasearch needs}

The efficient and specific ferredoxin-dependent repair system described above seems to be restricted to a limited number of extradiol dioxygenases, essentially in Proteobacteria. This system is beneficial to host cells in that it extends the range of aromatic substrates they can exploit (Polissi and Harayama 1993). Therefore, genetic transfer of xylT or one of its analogues into bacterial degraders other than Proteobacteria might be a valuable a strategy to improve the degradation efficiency of aromatic pollutants. Alternatively, search for bacterial strains producing extradiol dioxygenases more resistant to oxidative inactivation may lead to degraders with improved capabilities.

Acknowledgments : I thank C. Meyer for technical assistance and helpful discussions, and J. C. Willison for critical reading of the manuscript

\section{References}

Bartels I, Knackmuss HJ, Reineke W (1984) Suicide inactivation of catechol 2,3-dioxygenase from Pseudomonas putida mt-2 by 3-halocatechols. Appl. Environ. Microbiol. 47:500-505

Cerdan P, Wasserfallen A, Rekik M, Timmis KN, Harayama S (1994) Substrate specificity of catechol 2,3-dioxygenase encoded by TOL plasmid pWW0 of Pseudomonas putida and its relationship to cell growth. J. Bacteriol. 176:6074-6081

Dai S, Vaillancourt FH, Maaroufi H, Drouin N, Neau DB, Snieckus V, Bolin JT, Eltis LD (2002) Identification and analysis of a bottleneck in PCB biodegradation. Nat. Struct. Biol. 9:934-939

Ge Y, Vaillancourt FH, Agar NY, Eltis LD (2002) Reactivity of toluate dioxygenase with substituted benzoates and dioxygen. J. Bacteriol. 184:4096-4103 
Göbel M, Kranz OH, Kaschabek SR, Schmidt E, Pieper DH, Reineke W. (2004)

Microorganisms degrading chlorobenzene via a meta-cleavage pathway harbor highly similar chlorocatechol 2,3-dioxygenase-encoding gene clusters. Arch. Microbiol. 182, 147-156

Han S, Eltis LD, Timmis KN, Muchmore SW, Bolin JT (1995) Crystal structure of the biphenyl-cleaving extradiol dioxygenase from a PCB-degrading pseudomonad. Science 270:976-980

Harayama S, Kok M, Neidle EL (1992) Functional and evolutionary relationships among diverse oxygenases. Annu. Rev. Microbiol. 46:565-601

Harayama S, Rekik M (1990) The meta cleavage operon of TOL degradative plasmid pWW0 comprises 13 genes. Mol. Gen. Genet. 221:113-120

Hugo N, Armengaud J, Gaillard J, Timmis KN, Jouanneau Y (1998) A novel [2Fe-2S] ferredoxin from Pseudomonas putida mt-2 promotes the reductive reactivation of catechol 2,3-dioxygenase. J. Biol. Chem. 273:9622-9629

Hugo N, Meyer C, Armengaud J, Gaillard J, Timmis KN, Jouanneau Y (2000) Characterization of three XylT-like [2Fe-2S] ferredoxins associated with catabolism of cresols or naphthalene: Evidence for their involvement in catechol dioxygenase reactivation. J. Bacteriol. 182:5580-5585

Kaschabek SR, Kasberg T, Muller D, Mars AE, Janssen DB, Reineke W (1998) Degradation of chloroaromatics: purification and characterization of a novel type of chlorocatechol 2,3-dioxygenase of Pseudomonas putida GJ31. J. Bacteriol. 180:296-302

Kita A, Kita S, Fujisawa I, Inaka K, Ishida T, Horiike K, Nozaki M, Miki, K (1999) An archetypical extradiol-cleaving catecholic dioxygenase: the crystal structure of catechol 2,3-dioxygenase (metapyrocatechase) from Pseudomonas putida mt-2. Structure 7:25-34

Kovaleva EG, Lipscomb JD (2007) Crystal structures of $\mathrm{Fe} 2+$ dioxygenase superoxo, alkylperoxo, and bound product intermediates. Science 316:453-457

Mars AE, Kasberg T, Kaschabek SR, van Agteren MH, Janssen DB, Reineke W (1997) Microbial degradation of chloroaromatics: use of the meta-cleavage pathway for mineralization of chlorobenzene. J. Bacteriol. 179:4530-4537.

McLeod MP, Warren RL, Hsiao WWL, Araki N, Myhre M, Fernandes C, Miyazawa D, Wong W, Lillquist AL, Wang D, Dosanjh M, Hara H, Petrescu A, Morin RD, Yang G, Stott JM, Schein JE, Shin H, Smailus D, Siddiqui AS, Marra MA, Jones SJM, Holt R, Brinkman FSL, Miyauchi K, Fukuda M, Davies JE, Mohn WW, Eltis LD (2006) The complete genome of Rhodococcus sp. RHA1 provides insights into a catabolic powerhouse. Proc. Natl. Acad. Sci. U S A 103:15582-15587

Pinyakong O, Habe H, Omori $\mathrm{T}$ (2003) The unique aromatic catabolic genes in sphingomonads degrading polycyclic aromatic hydrocarbons (PAHs). J. Gen. Appl. Microbiol. 49:1-19

Polissi A, Harayama S (1993) In vivo reactivation of catechol 2,3-dioxygenase mediated by a chloroplast-type ferredoxin: a bacterial strategy to expand the substrate specificity of aromatic degradative pathways. EMBO J. 12:3339-3347

Riegert U, Burger S, Stolz A (2001) Altering catalytic properties of 3-chlorocatecholoxidizing extradiol dioxygenase from Sphingomonas xenophaga BN6 by random mutagenesis. J. Bacteriol. 183:2322-2330

Shu L, Chiou YM, Orville AM, Miller MA, Lipscomb JD, Que L, Jr. (1995) X-ray absorption spectroscopic studies of the Fe(II) active site of catechol 2,3-dioxygenase. Implications for the extradiol cleavage mechanism. Biochemistry 34:6649-6659 
Tropel D, Meyer C, Jouanneau Y (2002) Ferredoxin-mediated reactivation of the chlorocatechol 2,3-dioxygenase from Pseudomonas putida GJ31. Arch. Microbiol. 177:345-351

Vaillancourt FH, Bolin JT, Eltis LD (2006) The ins and outs of ring-cleaving dioxygenases. Crit. Rev. Biochem. Mol. Biol. 41:241-267

Vaillancourt FH, Labbe G, Drouin NM, Fortin PD, Eltis LD (2002) The mechanism-based inactivation of 2,3-dihydroxybiphenyl 1,2-dioxygenase by catecholic substrates. J. Biol. Chem. 277:2019-2027 


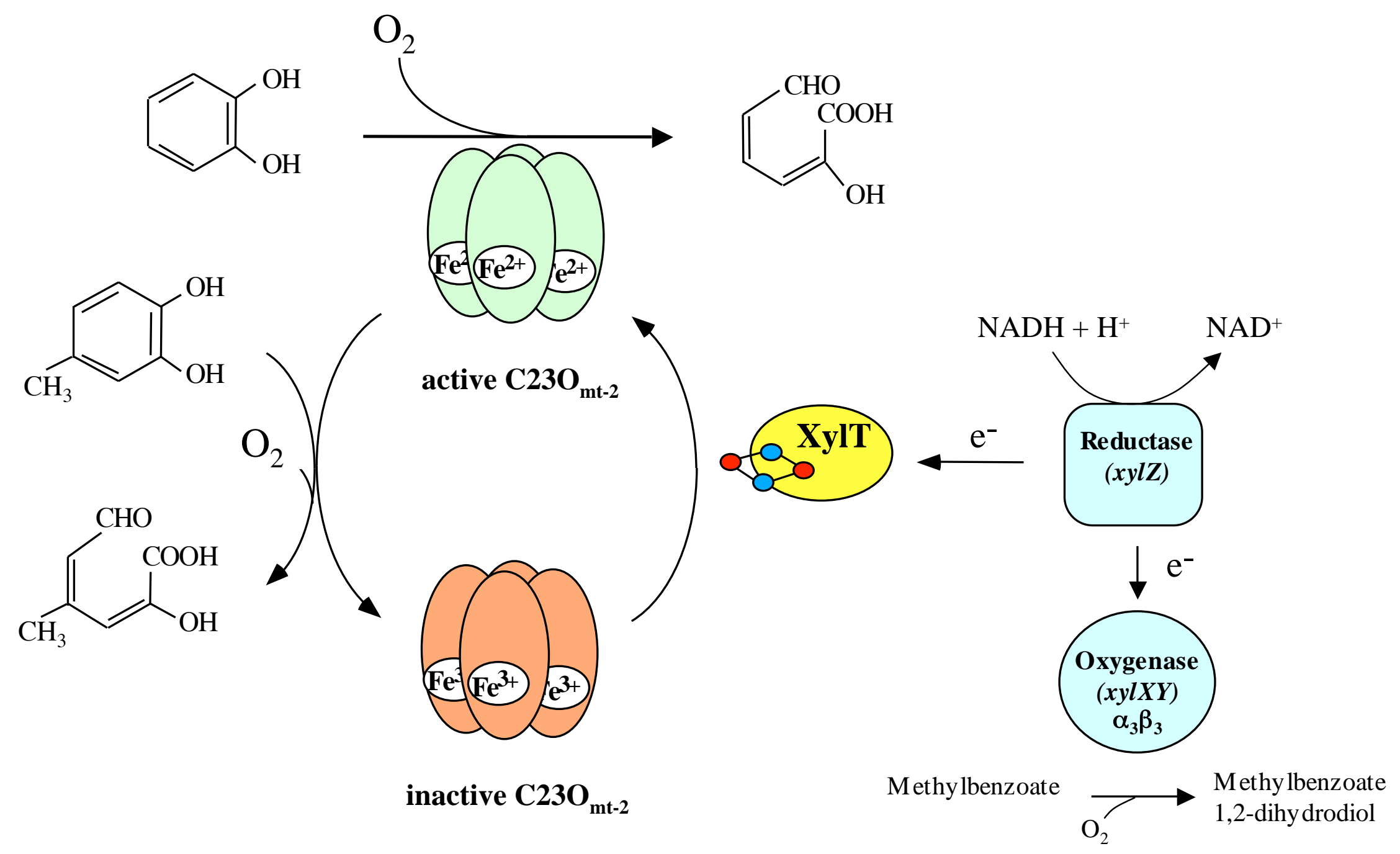

Oxidative inactivation of ring-cleavage extradiol dioxy genase, fig 2.TIF 